\title{
Power Controlled Network Protocols for Multi-Rate Ad Hoc Networks
}

\author{
Pan Li, Qiang Shen, Yuguang Fang, and Hailin Zhang
}

\begin{abstract}
In this paper, we propose for Multi-Rate ad hoc networks a cross-layer design using Power Control, called MRPC. MRPC consists of two parts. First, we propose a Multi-Rate Power Controlled MAC protocol, called MRPC-MAC. By carefully controlling the transmission power, it can enable concurrent transmissions, which is otherwise impossible for the IEEE 802.11 standard. Second, we propose a Multi-Rate Power Controlled Routing protocol, called MRPC-Routing. Different from traditional routing protocols, MRPC-Routing is not intended to find end-to-end paths, rather, it determines the next hop right before transmitting packets at the MAC layer. In this protocol, it uses the Effective Transport Capacity as the routing metric such that short links with high bandwidth are preferred and more concurrent transmissions can be enabled. Having these coupled power controlled MAC protocol and routing protocol, MRPC can greatly improve the spatial reuse and the network throughput. Simulation results also show MRPC-MAC, MRPC-Routing, and especially MRPC, can improve the network throughput significantly.
\end{abstract}

Index Terms-Wireless ad hoc networks; cross layer design; transmission power control.

\section{INTRODUCTION}

W IRELESS ad hoc networks consist of nodes communicating with each other via wireless medium directly or indirectly with the help of other nodes. They have gained more and more popularity due to easy and quick deployment with low cost. Recently, power control has been studied in ad hoc networks ([12][14][19][20][21]). It is shown that using power control can improve both the performance of MAC (Medium Access Control) protocols and that of routing protocols.

In addition to MAC protocol and routing protocol, the interaction between them also has significant impacts on the network performance. Thus, a cross-layer design is greatly needed for ad hoc networks. Especially, when power control is employed, how to make power controlled MAC and power controlled routing protocols cooperate to provide better network performance is an important and nontrivial problem.

Manuscript received April 28, 2008; revised August 5, 2008; accepted November 9, 2008. The associate editor coordinating the review of this paper and approving it for publication was J. Zhang.

P. Li and Y. Fang are with the Department of Electrical and Computer Engineering, University of Florida, Gainesville, Florida 32611-6130, Y. Fang is also with the National Key Laboratory of Integrated Services Networks, Xidian University, Xi'an, China (e-mail: \{lipanleo@, fang@ece\}ufl.edu).

Q. Shen is with the Provincial Key Lab of Information Coding and Transmission, Southwest Jiaotong University, Chengdu, China 610031 (email: q.shen@ufl.edu).

H. Zhang is with the National Key Laboratory of Integrated Services Networks, Xidian University, Xi'an, China (e-mail: hlzhang@xidian.edu.cn).

This work was supported in part by the U.S. National Science Foundation (NSF) under grant CNS-0721744 and grant DBI-0529012. The work of Y. Fang and $\mathrm{H}$. Zhang was also partially supported by the 111 Project under B08038 with Xidian University, China.

Digital Object Identifier 10.1109/TWC.2009.080580
Moreover, wireless ad hoc networks support both singlerate and multi-rate transmissions. Having variable transmission ranges, physical carrier sensing ranges, and SINRs (Signal-toInterference and Noise Ratio) for different transmission rates, multi-rate transmissions make the network endure constant topology changes, and hence make the cross layer design a more challenging design task.

In this paper, we propose for Multi-Rate ad hoc networks a new Power Controlled cross layer design, called MRPC, which is composed of two parts.

First, we propose a Multi-Rate Power Controlled MAC protocol, called MRPC-MAC, that can work in the context of many multi-rate schemes like ARF[13], RBAR[10], OAR[26]. In this study, we choose ARF as the basic multi-rate scheme since it is widely used by many IEEE 802.11 compliant wireless cards[2]. Then, based on that, we propose to control the transmission power to further improve the network throughput.

Basically, in IEEE 802.11 MAC, when one node is transmitting, the other nodes in its physical carrier sensing range should keep silent to avoid interference. Thus, even those transmissions that will not interfere with the ongoing one are still blocked. However, in MRPC-MAC, when one node overhears other nodes' transmissions, based on some information from those nodes indicating their tolerable interference level, the current node can be allowed to carry out its own transmissions by carefully controlling its transmission power. Thus, in MRPC-MAC, several concurrent transmissions can be enabled. The spatial reuse of the network can in turn be improved and hence the network throughput can be enhanced.

Second, we propose a Multi-Rate Power Controlled Routing protocol, called MRPC-Routing. As we all know, some commonly used routing protocols like AODV (Ad Hoc Ondemand Distance Vector) tend to find a path with minimum number of hops, which means the distance of each hop could be very large, and hence the tolerable interference level of each transmission could be very small. Thus, in the network, concurrent transmissions may not always be allowed, and the throughput improvement introduced by MRPC-MAC might be limited. In this paper, the proposed MRPC-Routing is a position-based routing (or geographic routing) protocol, where each node knows its neighboring nodes' locations and the destination's location. In MRPC-Routing, we use the product of successful data delivery ratio, bandwidth and distance progress along the direction to the destination as the routing metric, which we define as the Effective Transport Capacity. Each node always chooses the neighboring node which makes this metric largest as the next hop. By doing this, those short links with high bandwidth are preferred, which makes 
the transmissions tolerate more interference, and hence more concurrent transmissions can be enabled.

Moreover, different from traditional routing protocols which find end-to-end paths before transmitting packets, the proposed MRPC-Routing is not intended to find end-to-end paths. Instead, it determines the next hop right before transmitting packets at the MAC layer. In traditional routing protocols, each node determines the next hop by checking its routing table. Then, the packets are placed in a queue waiting for transmission at the MAC layer. Thus, these routing protocols are insensitive to the changes of channel conditions due to the queueing delay. In contrast, MRPC-Routing chooses the next hop for packets at the MAC layer, right before transmitting the packets, according to the channel conditions and the transmission power this node has at that moment. Thus, MRPC-Routing can adapt to the channel environments quickly.

The rest of this paper is organized as follows. In Section II, we review the related work. Section III and Section IV detail our proposed MRPC-MAC protocol, and MRPC-Routing protocol, respectively. Simulation results are presented in Section $\mathrm{V}$. We finally conclude this paper in Section VI.

\section{RELATED WORK}

In this section, we present the related work on power controlled MAC, power controlled routing, and cross layer design methodology, respectively.

\section{A. Power Controlled MAC}

Power controlled MAC protocols can be generally classified into two types. Many of them are to save energy in networks, like those in [12][14]. The others are to improve the network throughput. In this paper, we aim at enhancing network throughput by using power control.

In [19][20], two channels and two transceivers are used to improve the network throughput, which unfortunately introduce additional hardware cost and implementation complexity. Ding et al. [7] and Jia et al.[11] propose DEMAC and $\delta$-PCS , respectively, to improve the network throughput using a single channel and a single transceiver. But, they could only achieve limited improvement.

Muqattash et al. [21] also propose a throughput-oriented MAC protocol with a single channel and a single transceiver, called POWMAC. Different from the above protocols, POWMAC uses a new decision rule: when a node overhears other nodes' transmissions, it is still allowed to carry out its own DATA transmission as long as it does not interfere with the ongoing ones. Thus, according to POWMAC, several transmissions can happen concurrently. However, POWMAC introduces additional signalling overhead since an ongoing transmission needs to exchange $N(N>1)$ more RTS/CTS to enable $N$ concurrent transmissions. Besides, concurrent transmissions in POWMAC may not take place as expected if they are not well synchronized due to propagation delay.

In our previous work [18], utilizing a single channel and a single transceiver, we propose an adaptive transmission power controlled MAC protocol (ATPMAC) which only needs one RTS/CTS exchange for $N(N>1)$ concurrent transmissions.
ATPMAC also addresses the synchronization problem caused by propagation delay. It has been shown that ATPMAC can further improve the network throughput by more than $30 \%$ compared to POWMAC in a random multi-hop network. Notice that all the works mentioned above are proposed when only a single data rate is available. In this paper, we improve ATPMAC to make it work properly when multiple data rates are used for transmissions, resulting in the proposed MRPCMAC.

\section{B. Power Controlled Routing}

In the literature, many power controlled routing protocols such as [3][28] attempt to find paths which can save energy in the network. Usually, they first find an end-to-end path, determine the next hop, place packets in the queue, and then transmit at the MAC layer. Due to the queueing delay, the next hop determined earlier may not be appropriate any more when the packets are actually transmitted at the MAC layer. Especially, when power control is employed at the MAC layer, both the channel environment and the transmission capability of nodes, i.e., the transmission power, change constantly. Thus, we need to carefully choose the next hop at the MAC layer, right before transmitting the packets, which we call MAC layer routing. Unfortunately, it is very difficult to achieve this goal based on conventional routing protocols. In this paper, we propose a new routing protocol called MRPC-Routing based on geographic routing ([15] [16] [17] [22]), where we assume as usual each node knows the locations of its one-hop neighbors and the destination node. When a packet arrives at the MAC layer of a node, we choose its neighboring node that can make the Effective Transport Capacity largest as the next hop.

\section{Cross Layer Design}

Cross-layer design is crucial to the efficiency of wireless networks. Many papers formulate cross-layer design as an optimization problem, like [8] [6] [27] [30]. Different from them, this paper presents a cross-layer design by proposing coupled MAC protocol and routing protocol with power control for multi-rate ad hoc networks, i.e., the MRPC scheme.

\section{The Proposed MAC Protocol: MRPC-MAC}

In this section, we propose a single-radio, single-channel, and multi-rate MAC protocol to improve the spatial reuse by controlling the transmission power so that multiple transmissions can be enabled at the same time without interfering with each other. This MAC protocol is called Multi-Rate Power Controlled MAC, i.e., MRPC-MAC. The idea here is to allow a new transmission as long as it does not interfere with the ongoing transmission.

\section{A. Supporting Multi-rate Transmissions}

MRPC-MAC is based on IEEE 802.11 MAC proto$\mathrm{col}$, and it also follows the four-way handshake procedure (RTS/CTS/DATA/ACK) to carry out transmissions. In this subsection, we present how it supports multi-rate communication. 
TABLE I

INFORMATION OF NEIGHBORING NODES.

\begin{tabular}{|l|l|l|l|l|l|} 
Node ID & $P_{\min , 1}$ & $\ldots$ & $P_{\min , M}$ & Max Power $\left(P_{\max }\right)$ & FTime \\
\hline
\end{tabular}

As we mentioned before, MRPC-MAC can work in the context of many multi-rate schemes, such as ARF[13], RBAR[10], RRAA[29], and OAR[26]. Since many IEEE 802.11b wireless cards provide variable transmission rates according to ARF (Automatic Rate Fallback) scheme, we choose it as the basic multi-rate scheme in this study.

In ARF, control frames are sent at basic data rate denoted by $R_{\text {basic }}$, i.e., $1 \mathrm{Mbps}$ in IEEE $802.11 \mathrm{~b}$, and data frames are sent at a data rate chosen from a set of available data rates according to MAC transmission failures or successes. Specifically, for the second ACK miss, i.e., transmission failure, following successful transmissions, the data rate is reduced to the next lower level and a timer is set up. The data rate increases to the next higher level either when the timer expires or when the number of consecutive successful transmissions reaches ten.

Assume node $j$ receives signals from node $i$ at a power level of $P_{r}^{i}$. Let $R X_{t h, l}$ denote the receiver sensitivity for supporting data rate $R_{l}$, where $1 \leq l \leq M^{1}$. Then, the highest achievable data rate for successful DATA transmissions between node $i$ and node $j$, denoted by $R_{i j}$, can be determined by

$$
R_{i j}= \begin{cases}0 & \text { if } P_{r}^{i}<R X_{t h, 1} \\ R_{l} & \text { if } R X_{t h, l} \leq P_{r}^{i}<R X_{t h, l+1}, l \in[1, M-1] \\ R_{M} & \text { if } P_{r}^{i} \geq R X_{t h, M}\end{cases}
$$

\section{B. Allowing Concurrent Transmissions by Power Control}

In this subsection, we show how MRPC-MAC controls the transmission power so that multiple concurrent transmissions can be enabled.

As shown in Table I, each node maintains a table to keep some information of their neighboring nodes. "Node ID" is the MAC address of a neighboring node. $P_{\min , l}(1 \leq l \leq M)$ is the minimum transmission power required to successfully send a packet at a data rate of $R_{l}$ to that neighboring node when it does not suffer from any other interferences. "Max Power", denoted by $P_{\max }$, is the maximum transmission power allowed for the current node keeping this table to transmit packets without interfering with this neighboring node's ongoing transmission. "FTime" is the time that the neighboring node will finish its ongoing transmission. Each time a node overhears a packet from one of its neighboring nodes, it updates this table. The details on how to update this table will be discussed later.

1) Exchanging RTS/CTS: Recall the power propagation model given in [24], i.e.,

$$
P_{r}(d)=P_{t} \frac{C}{d^{\gamma}}
$$

${ }^{1}$ According to the IEEE 802.11 standards, in IEEE 802.11a, we have $M=$ $8,\left\{R_{l}: 1 \leq l \leq M\right\}=\{6,9,12,18,24,36,48,54\} \mathrm{Mbps}$, and in IEEE $802.11 \mathrm{~b}$, we have $M=4,\left\{R_{l}: 1 \leq l \leq M\right\}=\{1,2,5.5,11\}$ Mbps. where $P_{t}$ and $P_{r}$ are the transmitted power and the received power, respectively, $C$ is a constant related to the antenna profiles of the transmitter and the receiver, wavelength, and so on, $d$ is the distance between the transmitter and the receiver, and $\gamma$ is the path loss exponent.

When a receiver $j$ receives RTS from a transmitter $i$, it can collect the reception power $P_{r}^{i}$, and the transmission power $P_{t}^{i}$, which is a new field we add in RTS frames. Thus, from (2), we can obtain

$$
P_{r}^{i}=C \cdot \frac{P_{t}^{i}}{d_{i j}^{\gamma}}
$$

where $d_{i j}$ is the distance between node $i$ and node $j$.

Since $R X_{t h, l}$ is the receiver sensitivity for supporting data rate $R_{l}$, then by assuming the physical channel is symmetric, the minimum power required for the receiver $j$ to successfully transmit a packet to the transmitter $i$ at data rate $R_{l}$, i.e., $P_{\min , l}^{i}$ as mentioned before, satisfies

$$
R X_{t h, l}=C \cdot \frac{P_{m i n, l}^{i}}{d_{i j}^{\gamma}}
$$

From (3) and (4), we can get for any $1 \leq l \leq M$,

$$
P_{\text {min }, l}^{i}=\frac{P_{t}^{i} \cdot R X_{t h, l}}{P_{r}^{i}} .
$$

After obtaining $P_{m i n, l}^{i}$, the receiver $j$ checks in Table I to find those active neighboring nodes denoted by set $S$, i.e.,

$$
S=\left\{k \quad \mid \quad \text { FTime }^{k}>t_{\text {now }}\right\}
$$

where FTime $^{k}$ is the time that the neighboring node $k$ will finish its ongoing transmission, and $t_{\text {now }}$ is the current time. Notice that $S$ may change over time.

The maximum allowed transmission power of the receiver $j$, denoted by $P_{\text {allow }}^{j}$, is

$$
P_{\text {allow }}^{j}= \begin{cases}\min _{k \in S}\left\{P_{\max }^{k}\right\} & \text { if } S \neq \emptyset \\ P_{M A X} & \text { if } S=\emptyset\end{cases}
$$

where as we mentioned before, $P_{\max }^{k}$ is the maximum transmission power of node $j$ at which $j$ 's transmission will not interfere with $k$ 's, $\emptyset$ stands for the empty set, and $P_{M A X}$ is the maximum allowed transmission power of all the nodes. We will present how to obtain $P_{\max }^{k}$ shortly.

If $P_{\text {allow }}^{j}$ is less than $P_{m i n, 1}^{i}$, then the receiver $j$ is not allowed to reply with CTS because this CTS will be definitely not received by the transmitter. Otherwise, CTS is transmitted after a period of SIFS with the transmission power $P_{\text {allow }}^{j}$. Thus, this CTS transmission will not interfere with $j$ 's active neighboring nodes' transmissions, and it is possible that this CTS could be correctly received.

The same as RTS frame, our CTS frame also contains the transmission power. Since the CTS frame defined in IEEE 802.11 standard only has the MAC address of the frame's receiver, we add a new field called "Transmitter Address" in our CTS frame to put in the MAC address of the frame's transmitter. By doing this, other nodes overhearing CTS from the receiver $j$ can update their information about $j$ kept in Table I. We will introduce this process in the next subsection. 
2) Overhearing CTS: We also add another new field called "Interference Level" in our CTS frame, which is the maximum average interference level each neighboring node is allowed to generate to the receiver $j$. Denote "Interference Level" by $P_{\text {interf }}$, then we obtain

$$
P_{\text {interf }}^{j}=\frac{\frac{P_{r}^{i}}{S I N R_{l}}-P_{\text {noise }}}{N \cdot(1+\beta)}=\frac{P_{r}^{i}-S I N R_{l} \cdot P_{\text {noise }}}{N \cdot(1+\beta) \cdot S I N R_{l}}
$$

where $l$ is included in RTS frame indicating the data rate $R_{l}$ the transmitter has chosen, $S I N R_{l}(1 \leq l \leq M)$ is the signalto-interference plus noise ratio required to support the data rate, $P_{\text {noise }}$ is the noise power level at the receiver, $N$ is the number of the neighboring nodes of the receiver $j$, which can be obtained by checking the number of nodes in Table I, and $\beta(\beta>0)$ indicates the interference caused by the nodes out of the transmission range, which is about 0.5 for the two-ray propagation model and uniformly distributed terminals [25].

After CTS is sent out, some neighboring nodes of the receiver $j$ may overhear it and hence can update their information about node $j . P_{\min , l}^{j}$ is calculated similarly to (5), i.e.,

$$
P_{\min , l}^{j}=\frac{P_{t}^{j} \cdot R X_{t h, l}}{P_{r}^{j}} .
$$

Next time when a neighboring node $k$ wants to send packets to node $j$, it can carry out the transmission at a data rate $R_{l}$ chosen according to ARF scheme only if its maximum allowed transmission power, $P_{\text {allow }}^{k}$, is no smaller than $P_{\min , l}^{j}$.

Since this CTS contains the transmission power of receiver $j$, denoted by $P_{t}^{j}$, for a neighboring node $k$, we have

$$
P_{r}^{j}=C \cdot \frac{P_{t}^{j}}{d_{j k}^{\gamma}},
$$

and

$$
P_{\text {interf }}^{j}=C \cdot \frac{P_{\max }^{j}}{d_{j k}^{\gamma}} .
$$

where $d_{j k}$ denotes the distance between receiver $j$ and the neighboring node $k$, and $P_{\max }^{j}$ is the maximum transmission power allowed for node $k$ to transmit packets without affecting the reception of the following DATA frames at receiver $j$. From (9) and (10), we obtain

$$
P_{\text {max }}^{j}=\frac{P_{\text {interf }}^{j} \cdot P_{t}^{j}}{P_{r}^{j}} .
$$

After successfully overhearing the CTS from node $j$, a neighboring node $k$ will update the "FTime" field in Table I for node $j$ by setting it to the current time plus NAV(Network Allocation Vector) duration if NAV is set and to the current time if not. The NAV is set according to the following rules. If node $k$ does not want to send out any frames, it does not set its NAV. Because with $P_{\text {allow }}^{j}$ defined in (6), even later it has some frames to transmit, those transmissions will not interfere with $j$ 's reception. Or, if node $k$ has a DATA frame for node $j$, it will set its NAV in the same way as that defined in IEEE 802.11 standard. If the neighboring node $k$ has a DATA frame for some node $m$ other than node $j$, it will also set its NAV if $P_{\text {allow }}^{k}<P_{m i n, l}^{m}$, i.e., the maximum transmission power of node $k$ is smaller than the minimum transmission power required to transmit frames to node $m$ at the data rate $R_{l}$ chosen by ARF scheme. Otherwise, i.e., if $P_{\text {allow }}^{k} \geq P_{m i n, l}^{m}$, node $k$ does not set its NAV and will transmit its own DATA frame after a period of SIFS. Thus, there is a good chance that some neighboring nodes of receiver $j$ can transmit DATA frames at the same time as node $i$ without affecting node $j$ 's DATA reception, and hence the spatial reuse can be significantly improved.

3) Overhearing RTS: We add a new field in our ACK frames called "Transmission Power" to place in the transmission power of the ACK frames. So, when node $i$ receives an ACK from node $j$, it can obtain the reception power $P_{r}^{j}$, as well as the transmission power $P_{t}^{j}$ of the ACK frame. Thus, node $i$ can calculate the maximum average interference level each neighboring node is allowed to node $i$ for its next DATA transmission, denoted by $P_{\text {interf }}^{i}$, in a similar way to (7), i.e.,

$$
P_{\text {interf }}^{i}=\frac{P_{r}^{j}-S I N R_{l} \cdot P_{\text {noise }}}{N \cdot(1+\beta) \cdot S I N R_{l}} .
$$

where $l$ corresponds to the data rate $R_{l}$ determined by ARF. Besides, node $i$ also updates $P_{\text {min }, l}^{j}$ in Table I according to (8). Next time when node $i$ has a RTS frame to transmit, it will put $P_{t}^{i}$ and $P_{\text {interf }}^{i}$ in two new fields of the RTS frame, i.e., "Transmission Power" field and "Interference Level" field, respectively. Any neighboring node that overhears this RTS will update their $P_{m i n, l}^{i}, P_{\text {max }}^{i}$, and FTime $i$ in Table I accordingly, in a similar way to what we described before. Particularly, if $P_{\text {allow }}^{k} \geq P_{m i n, l}^{m}$, node $k$ does not set its NAV and will carry out its DATA transmission a period of $2 * S I F S+\frac{L_{C T S}}{R_{b a s i c}}$ later, where $L_{C T S}$ is the length of a CTS frame and $R_{\text {basic }}$ is the basic rate. Thus, there is a good chance that some neighboring nodes of transmitter $i$ can transmit DATA frames at the same time as node $i$ without affecting node $i$ 's ACK reception, and the spatial reuse can be significantly improved.

Moreover, when combining the operations after overhearing RTS and those after overhearing CTS, we should consider a special case. Assume a node overhears a RTS frame and is allowed to carry out its DATA transmission. If later this node overhears a CTS frame and is not allowed to carry out the transmission any more, then it does not carry out the transmission as planned and waits until the channel is idle.

4) Tuning the Physical Carrier Sensing Threshold: IEEE 802.11 standard defines two important concepts: transmission range and physical carrier sensing range, which are determined by receiver sensitivity and physical carrier sensing threshold, respectively. Two nodes within the transmission range of each other can communicate directly, and two nodes within the physical carrier sensing range of each other cannot transmit frames at the same time. Different data rates have different transmission ranges and different physical carrier sensing ranges.

In [18], we have shown that physical carrier sensing range needs to be the same as transmission range in order for the proposed ATPMAC to work efficiently. Similarly, in this study, we also propose to set physical carrier sensing threshold to receiver sensitivity for each available data rate such that the carrier sensing ranges are the same as the transmission ranges. Besides, we place the data rate chosen for DATA transmissions 
in both RTS and CTS frames. The nodes overhearing RTS or CTS carry out their transmissions at the same transmission rate as the ongoing one.

5) More Discussions: As we discussed in Section III-B2 and Section III-B3, one node overhearing CTS or RTS will wait for a period of $S I F S$ or $\frac{L_{C T S}}{R_{\text {basic }}}+2 * S I F S$, respectively, to initiate a DATA transmission at the same time as the current transmitter does. However, taking the propagation delay into consideration, this synchronization is very difficult to achieve and concurrent transmissions may not be enabled. As we pointed out in [18], this problem can be addressed by employing an advanced physical layer technology that can correctly detect and capture a strong frame during the reception of a weak frame. One such an example is Lucent's physical layer (PHY) design with "Message-In-A-Message" (MIM) support [4]. Furthermore, as we explained in [18], the concurrent transmissions planned by neighboring nodes after overhearing RTS/CTS might fail. In this case, the neighboring nodes do not double their contention windows as required by IEEE 802.11 for the fairness issue.

\section{The Proposed Routing Protocol: MRPC-ROUTING}

In Section III, we have introduced our proposed MRPCMAC. In this protocol, the neighboring nodes of a transmitter or a receiver engaged in a transmission are allowed to carry out their own transmissions if the ongoing transmission can tolerate more interference. Thus, the spatial reuse can be improved and hence the throughput can be enhanced. However, we should also notice that how efficiently MRPCMAC can perform depends on the design of routing protocols. For example, AODV (Ad Hoc On-demand Distance Vector) routing protocol tends to find a path with minimum number of hops, implying that the length of each hop could be very large, and hence the tolerable interference of each transmission could be small. Thus, concurrent transmissions may not always be allowed, and the throughput improvement might be limited. For another example, if a routing protocol always chooses the nearest neighboring node as the next hop, the number of endto-end hops may be very large and the probability that a packet is lost is very high. Thus, the network performance may be even worse.

From the above discussion, we can find that how to design a routing protocol that can cooperate with MRPC-MAC to improve the network throughput is a very important and nontrivial problem. In this section, we detail the proposed routing protocol, called MRPC-Routing.

MRPC-Routing is based on geographic routing, where we assume as usual each node knows the locations of its one-hop neighbors and the destination node. In geographic routing, there are several popular routing strategies, such as forwarding data packets to the neighbor geographically closest to the destination, or to the neighbor with the largest distance progress towards the destination. Obviously, these routing strategies find long links, which may not tolerate much more interference. Thus, concurrent transmissions can rarely happen. Different from those routing strategies, in MRPCRouting, a node determines for a packet the next hop, denoted

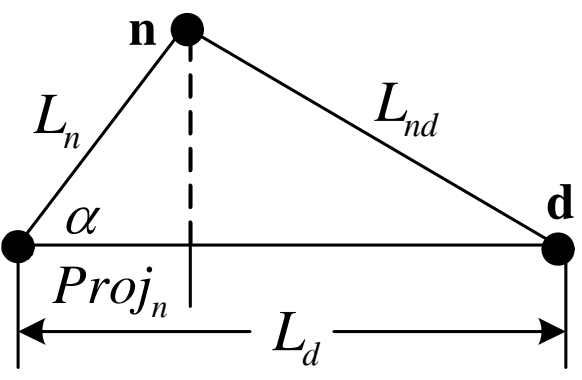

Fig. 1. The calculation of $\operatorname{Proj}_{n}$.

as $N E X T$, as follows:

$$
N E X T=\arg \max _{n \in S^{\prime}}\left\{\eta_{n} \cdot B_{n} \cdot \operatorname{Proj}_{n}\right\}
$$

where $S^{\prime}$ is the set of neighbors of the current node, $\eta_{n}, B_{n}$, and $\operatorname{Proj}_{n}$ are the successful data delivery ratio to neighbor $n$, the maximum bandwidth to neighbor $n$, and the distance progress along the direction to the destination, respectively. For convenience, we define the product of $\eta_{n}, B_{n}$, and $\operatorname{Proj}_{n}$ as the effective transport capacity of neighbor $n$, compared to the transport capacity proposed in [9]. According to (12), we always choose the node which can maximize the effective transport capacity to act as the next hop. Thus, those short links with high bandwidth may be preferred, and hence more concurrent transmissions can be supported and the spatial reuse can be improved.

In (12), $\eta_{n}$ can be easily obtained by monitoring the transmission at the MAC layer. $B_{n}$ can be obtained by

$$
B_{n}= \begin{cases}0 & \text { if } P_{\text {allow }}<P_{\text {min }, 1}^{n} \\ R_{l} & \text { if } P_{\text {min }, l}^{n} \leq P_{\text {allow }}<P_{\text {min }, l+1}^{n} \\ R_{M} & \text { if } P_{\text {allow }} \geq P_{\text {min }, M}^{n}\end{cases}
$$

where $1 \leq l \leq M-1, P_{\text {allow }}$ is the maximum allowed transmission power for the current node, and $P_{m i n, l}^{n}$ is the minimum transmission power for the current node to transmit to node $n$ at data rate $R_{l}$. Besides, $\operatorname{Proj}_{n}$ can be calculated as follows:

$$
\operatorname{Proj}_{n}=L_{n} \cdot \cos \alpha, \quad \cos \alpha=\frac{L_{n}^{2}+L_{d}^{2}-L_{n d}^{2}}{2 L_{n} L_{d}}
$$

where as shown in Fig. $1, L_{n}, L_{d}$, and $L_{n d}$ are the distances from current node to neighbor node $n$, from current node to destination, and from neighbor node $n$ to destination, respectively.

Notice that the set of the neighbors of a node $j$, denoted by $S_{j}^{\prime}$, may vary from time to time, which can be determined by

$$
S_{j}^{\prime}=\left\{m \quad \mid \quad P_{\text {allow }}^{j} \geq P_{m i n, 1}^{m}\right\}
$$

where as defined in Section III, $P_{\text {allow }}^{j}$ is the maximum allowed transmission power of node $j$ so that its transmissions will not interfere with its neighbors' ongoing transmissions, and $P_{m i n, 1}^{m}$ is the minimum transmission power for node $j$ to support the lowest data rate between node $j$ and node $m$.

Since $P_{\text {allow }}^{j}$ changes over the time, $S_{j}^{\prime}$ also changes. Many conventional routing protocols determine for packets the next hop at the routing layer, and then put them in the queue waiting 
for transmission at the MAC layer. Due to the queueing delay, $S_{j}^{\prime}$ may be different when one packet is about to be transmitted at the MAC layer from that when its next hop is determined at the routing layer. Thus, the previously determined next hop may not be the optimum one when the packet is ready to transmit. What is worse, it may not even be reached due to the reduced transmission power. In MRPC-Routing, we determine the next hop at the MAC layer, right before the packets are about to be transmitted. By doing this, the next hop can be determined based on the current channel environment and the current node's transmission capability, i.e., its transmission power.

Besides, (12) only gives a rule to determine the next hop when the destination is not within the transmission range of the current node. We still need to consider how to carry out the transmission when the destination is one of the one-hop neighbors of the current node. One method is to transmit packets directly to the destination. Unfortunately, it is possible that the destination is a little bit far away, and the bandwidth from the current node to the destination would be low. As we mentioned before, our routing strategy prefers short links with high bandwidth. Thus, this last-hop would become the bottleneck and many packets may be dropped. In order to address this problem, we choose the next hop as follows. The current node calculates for each of its neighboring nodes the minimum value among the bandwidth from current node to that neighboring node and the bandwidth from the same neighboring node to the destination. The neighboring node whose minimum value described above is the largest is selected as the next hop, i.e.,

$$
N E X T=\arg \max _{n \in S^{\prime}}\left\{\min \left\{B_{n}, B_{n d}\right\}\right\}
$$

where $B_{n}$ and $B_{n d}$ are the bandwidth from the current node to the neighboring node $n$, and the bandwidth from the neighboring node $n$ to the destination, respectively. The same as before, $B_{n}$ can be obtained from (13). We also estimate $B_{n d}$ according to (13), by assuming $P_{\text {allow }}^{n}=P_{M A X}$, the maximum allowed transmission power of all the nodes. Besides, we set $B_{d d}=R_{M}$.

Finally, it is worthwhile to mention that MRPC-Routing just proposes a new routing strategy and it may be integrated with many routing recovery schemes like those in [5] [15] [16].

\section{Simulation Results}

In this section, we use NS2 (version 2.29) to evaluate the performance of the proposed MRPC scheme. We first compare in Section V-A the performance of MRPC-MAC with that of ARF. Then, in Section V-B, we present the performance of MRPC-Routing with two different routing metrics. Finally, we evaluate the performance of MRPC scheme, i.e., MRPCMAC together with MRPC-Routing in Section V-C. Some of our simulation parameters are shown in Table II[1].

\section{A. Performance of MRPC-MAC}

In order to show how well our proposed MRPC-MAC performs, we compare its performance with that when no power control is employed, i.e., the performance of ARF. Notice that in ARF, according to the protocol [13], the physical
TABLE II

SIMULATION PARAMETERS.

\begin{tabular}{|l|l|}
\hline Parameters & Value \\
\hline Channel frequency & $2.4 \mathrm{GHz}$ \\
Basic rate & $1 \mathrm{Mbps}$ \\
Available data rates & $1,2,5.5,11 \mathrm{Mbps}$ \\
Maximum transmission power & $15 \mathrm{dBm}$ \\
SINR threshold & $4,7,11,16 \mathrm{~dB}$ \\
Receiver sensitivity & $-94,-91,-87,-82 \mathrm{dBm}$ \\
Carrier sensing threshold & $-100 \mathrm{dBm}$ \\
Packet size & 1000 bytes \\
RTS retry limit & 7 \\
\hline
\end{tabular}

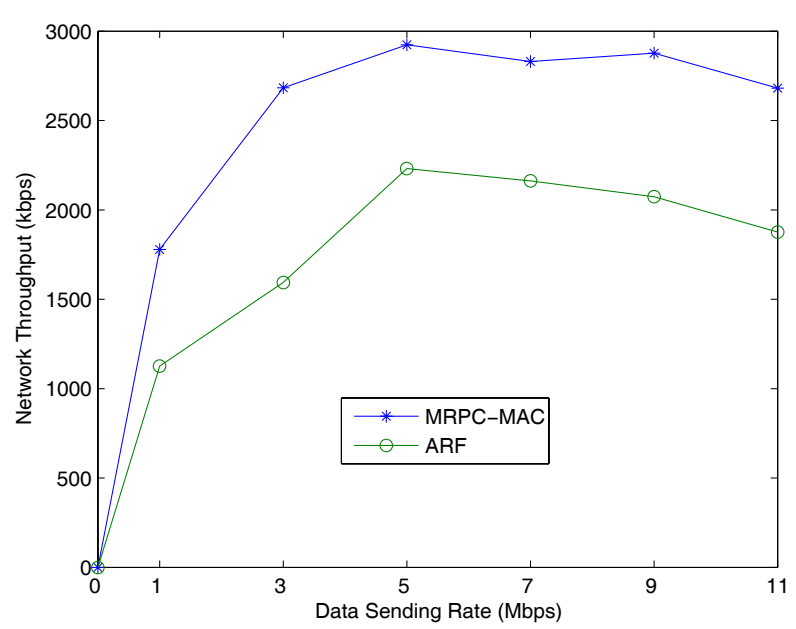

Fig. 2. The network throughputs of MRPC-MAC and ARF in a multi-hop scenario.

carrier sensing threshold is as shown in Table II, which is the same for different data rates. While in the MRPC-MAC, as we mentioned before, we set the physical carrier sensing thresholds to the same as the receiver sensitivities for different data rates, i.e., $-94,-91,-87,-82 \mathrm{dBm}$ for $1,2,5.5,11$ Mbps, respectively. Besides, recall that in ARF a timer will be set up when the data rate fallbacks to a lower rate. The timeout value was not explicitly specified in [13]. So, similar to [23], we use a virtual timer that accounts for the number of transmission attempts. As shown in [23], the performance of ARF is relatively insensitive to the choice of timeout value, and we set the timeout to 15 , the same as that in [23].

In our simulations, we randomly distribute 50 nodes in an area of $1000 \mathrm{mx} 1000 \mathrm{~m}$. The network uses AODV (Adhoc Ondemand Distance Vector) routing protocol. We run the simulation ten times and each time we set up ten random CBR (Constant Bit Rate) flows. The network throughput averaged over ten runs is shown in Fig. 2.

We can clearly see that MRPC-MAC constantly outperforms ARF, with improvements ranging from $14 \%$ to $65 \%$. The improvement of MRPC-MAC over ARF is contributed by concurrent transmissions.

\section{B. Performance of MRPC-Routing}

It is unfair to compare the performance of MRPC-Routing with that of AODV or other on-demand routing protocols since MRPC-Routing is a kind of geographic routing protocol which knows some position information of the nodes in the network while the latter routing protocols do not. So, in this 


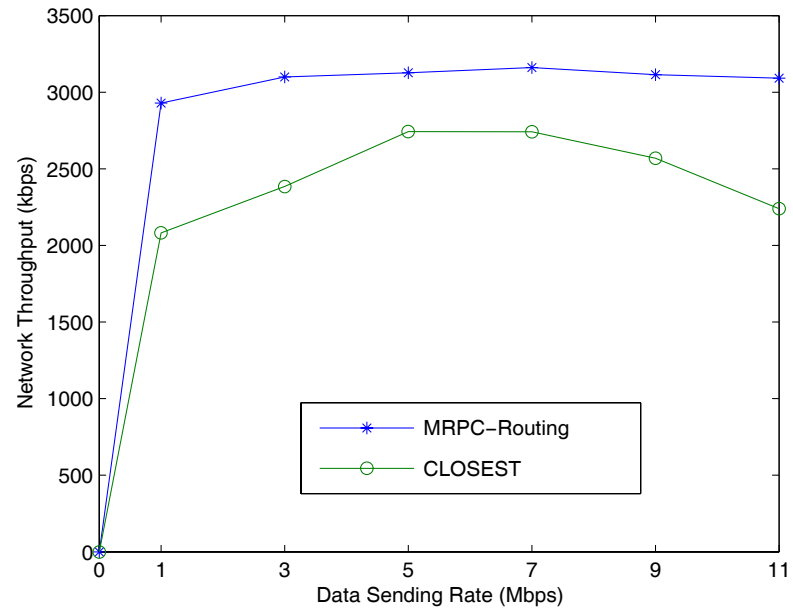

Fig. 3. The network throughputs of MRPC-Routing and CLOSEST in a multi-hop scenario.

subsection, we evaluate the performance of MRPC-Routing with two different routing metrics.

One routing metric is to forward packets to the neighbor with the largest effective transport capacity, i.e., the one used in MRPC-Routing as we introduced in Section IV. The other one is to forward packets to the neighbor closest to the destination, which is popularly used in geometric routing. We denote this routing metric by "CLOSEST".

In our simulations, we also randomly distribute 50 nodes in an area of $1000 \mathrm{mx} 1000 \mathrm{~m}$. The network uses ARF as the MAC protocol. We run the simulation ten times with ten random CBR (Constant Bit Rate) flows for each time. The network throughput averaged over ten runs is shown in Fig. 3.

With no surprise, we find that MRPC-Routing achieves better performance than CLOSEST by up to $40 \%$. This is because MRPC-Routing can find short links with high bandwidth while CLOSEST prefers the long links with low bandwidth.

\section{Performance of MRPC}

In this subsection, we evaluate the performance of our proposed cross-layer design scheme MRPC, i.e., MRPC-MAC working together with MRPC-Routing. We compare the performance of MRPC with that of ARF working with CLOSEST.

The same as above, we randomly distribute 50 nodes in an area of $1000 \mathrm{~m} \times 1000 \mathrm{~m}$. We run the simulation ten times with ten random CBR (Constant Bit Rate) flows each time. The network throughputs of MRPC and ARF with CLOSEST are averaged over ten runs and are shown in Fig. 4. We find that MRPC achieves significant improvement over ARF with CLOSEST. The network throughput of MRPC is about $40 \%$ to $70 \%$ higher than that of ARF with CLOSEST. Moreover, we further study the impacts of the number of nodes on the performance of MRPC. We change the number of nodes from 20 to 90 while keep the other network settings the same as above. The simulation results are shown in Fig. 5. We can observe that both the network throughput of MRPC and that of ARF with CLOSEST decrease as the number of nodes increases due to the increased interference. However, MRPC can achieve up to $92 \%$ higher throughput than ARF

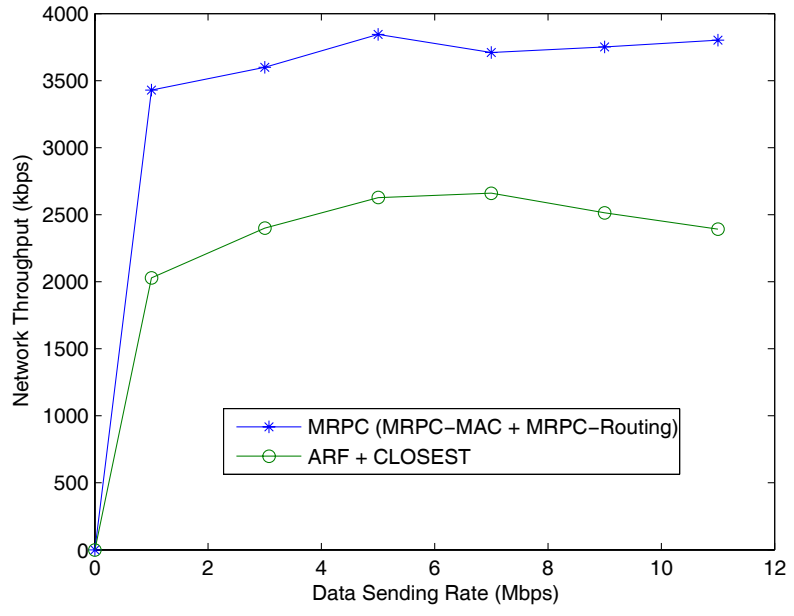

Fig. 4. The network throughput of MRPC and ARF with CLOSEST in a multi-hop scenario.

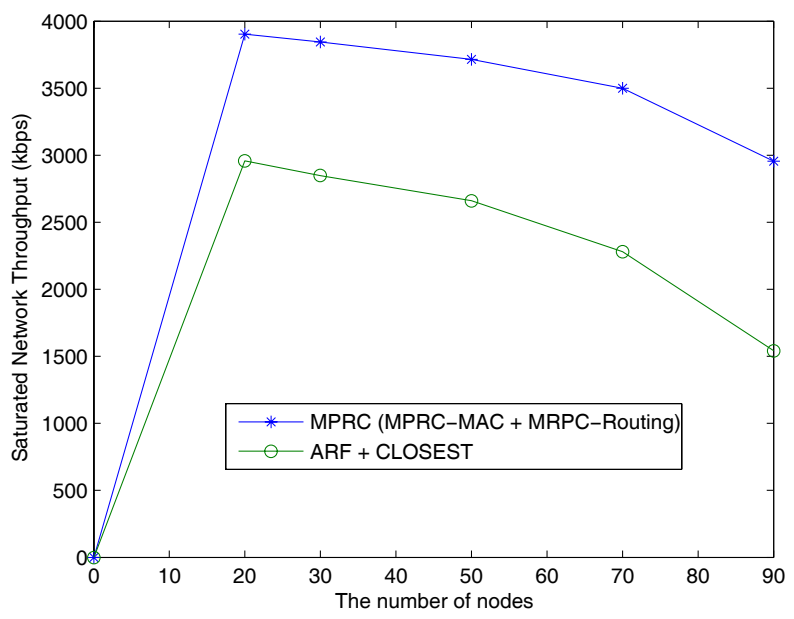

Fig. 5. The network throughput of MRPC and ARF with CLOSEST when the number of nodes ranges from 20 to 90 .

with CLOSEST because it can increase spatial reuse ratio by enabling more concurrent transmissions.

We also study the performance of MRPC in mobile ad hoc networks. We use the Random WayPoint (RWP) mobility model in the simulation. There are still 50 nodes in an area of $1000 \mathrm{~m} \times 1000 \mathrm{~m}$. We have ten runs and each time we set up 10 random CBR (Constant Bit Rate) flows. The averaged network throughputs of MRPC when the maximum speed is $0,1,3$, and $5 \mathrm{~m} / \mathrm{s}$ are shown in Fig. 6. The results show that the performance of MRPC degrades when the nodes' mobility become more active. The reason is that in MRPCMAC, we rely on power estimation to determine the tolerable interference level of ongoing transmissions and the minimum power for one node to reach another one, which becomes inaccurate when nodes are moving.

\section{CONCLUSION AND FutURE WORK}

In this paper, we propose a new power controlled crosslayer design, called MRPC, for multi-rate ad hoc networks. Simulation results show that the coupled MRPC-MAC and MRPC-Routing can work together well. Either of them, and 


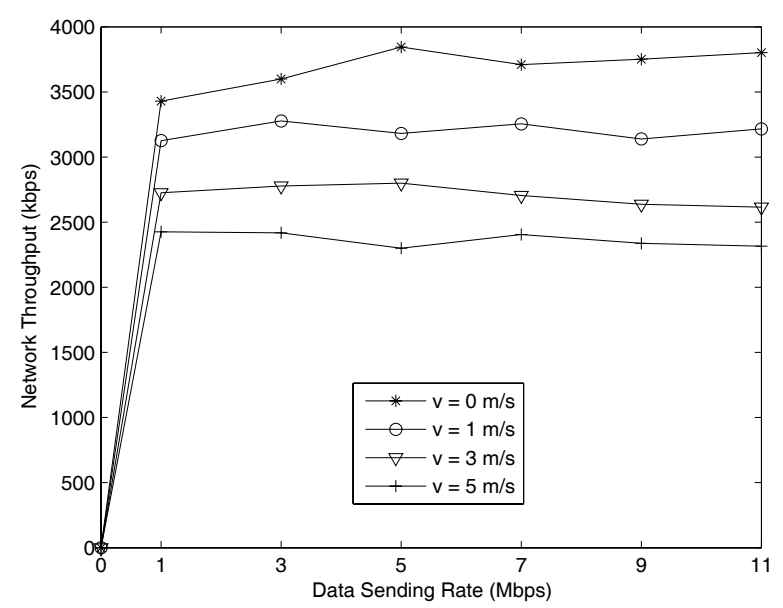

Fig. 6. The network throughputs of MRPC when the maximum speed of nodes are $0,1,3$, and $5 \mathrm{~m} / \mathrm{s}$.

especially these two working cooperatively, can significantly improve the network throughput.

However, we must realize that there are some limitations on MRPC. First, it does not proactively address the mobility issue. Simulation results indicate the performance of MRPC degrades when nodes are moving. Second, the routing metric used is shown to be good, but it may not be the optimal one. How to find an optimal path to maximize the end-toend throughput is still an open problem. We will investigate these issues in the future.

\section{REFERENCES}

[1] [Online] Available: http://www.cisl.columbia.edu/grads/ozgun/ProjectApplication.html.

[2] "Wireless LAN medium access control (MAC) and physical layer (PHY) specifications," IEEE Standards Working Group, 1999.

[3] P. Bergamo, D. Maniezzo, A. Travasoni, A. Giovanardi, G. Mazzini, and M. Zorzi, "Distributed power control for energy efficient routing in ad hoc networks," Wireless Networks, vol. 10, no. 1, pp. 29-42, 2004.

[4] J. Boer, H. Bokhorst, W. Diepstraten, A. Kamerman, R. Mud, H. Driest, and R. Kopmeiners, "Wireless LAN with enhanced capture provision," U.S. patent 5987033, Nov. 1999.

[5] P. Bose, P. Morin, I. Stojmenovic, and J. Urrutia, "Routing with guaranteed delivery in ad hoc wireless networks," Wireless Networks, vol. 7, no. 6, pp. 609-616, 2001.

[6] R. Cruz and A. Santhanam, "Optimal routing, link scheduling and power control in multi-hop wireless networks," in Proc. IEEE International Conf. Computer Commun. (INFOCOM'03), San Francisco, CA, USA, Ma. 2003.

[7] P. Ding, J. Holliday, and A. Celik, "Demac: an adaptive power control MAC protocol for ad-hoc networks," in Proc. IEEE 16th International Sym. Personal, Indoor Mobile Radio Commun. (PIMRC'05), Berlin, Germany, Sept. 2005.

[8] A. Eryilmaz and R. Srikant, "Joint congestion control, routing, and MAC for stability and fairness in wireless networks," IEEE J. Select. Areas Commun., vol. 24, no. 8, pp. 1514-1524, Aug. 2006.

[9] P. Gupta and P. Kumar, "The capacity of wireless networks," IEEE Trans. Inform. Theory, vol. 46, no. 2, pp. 388-404, Mar. 2000.
[10] G. Holland, N. Vaidya, and P. Bahl, "A rate-adaptive MAC protocol for multi-hop wireless networks," in Proc. ACM MobiCom, Rome, Italy, 2001.

[11] L. Jia, X. Liu, G. Noubir, and R. Rajaraman, "Transmission power control for ad hoc wireless networks: throughput, energy and fairness," in Proc. IEEE Wireless Commun. Networking Conf. (WCNC'05), New Orleans, LA, USA, Mar. 2005.

[12] E. Jung and N. Vaidya, "A power control MAC protocol for ad hoc networks," in Proc. ACM MobiCom, Atlanta, GA, USA, Sept. 2002.

[13] A. Kamerman and L. Monteban, "Wavelan II: a high-performance wireless LAN for the unlicensed band," Bell Labs Technical J., pp. 118-133, Summer 1997.

[14] P. Karn, "Maca: a new channel access method for packet radio," in Proc. 9th ARRL Computer Networking Conf., London, Ontario, Canada, 1990.

[15] B. Karp and H. Kung, "GPSR: greedy perimeter stateless routing for wireless networks," in Proc. ACM MobiCom, Boston, MA, USA, Aug. 2000.

[16] F. Kuhn, R. Wattenhofer, Y. Zhang, and A. Zollinger, "Geometric ad-hoc routing: of theory and practice," in Proc. ACM 22nd Annual Symposium Principles Distributed Computing (PODC 2003), Boston, MA, USA, July 2003.

[17] S. Lee, B. Bhattacharjee, and S. Banerjee, "Efficient geographic routing in multihop wireless networks," in Proc. ACM MobiHoc, UrbanaChampaign, IL, USA, May 2005.

[18] P. Li, X. Geng, and Y. Fang, "An adaptive power controlled MAC protocol for wireless ad hoc networks," to appear in IEEE Trans. Wireless Commun.

[19] J. Monks, V. Bharghavan, and W. Hwu, "A power controlled multiple access protocol for wireless packet networks," in Proc. IEEE International Conf. Computer Commun. (INFOCOM'01), Anchorage, AK, USA, Apr. 2001.

[20] A. Muqattash and M. Krunz, "Power controlled dual channel (PCDC) medium access protocol for wireless ad hoc networks," in Proc. IEEE International Conf. Computer Commun. (INFOCOM'03), San Francisco, CA, USA, Mar. 2003.

[21] A. Muqattash and M. Krunz, "Powmac: a single-channel power-control protocol for throughput enhancement in wireless ad hoc networks," IEEE J. Select. Areas Commun., vol. 23, no. 5, pp. 1067-1084, May 2005.

[22] D. Niculescu and B. Nath, "Trajectory based forwarding and its applications," in Proc. ACM MobiCom (MobiCom'03), Annapolis, MD, USA, June 2003.

[23] D. Qiao, S. Choi, and K. Shin, "Goodput analysis and link adaptation for ieee 802.11a wireless LANs," IEEE Trans. Mobile Computing, vol. 1, no. 4, pp. 278-292, 2002.

[24] T. Rappaport, Wireless Commun.: Principles and Practice, 2nd ed. Prentice-Hall PTR, 2002.

[25] T. Rappaport and L. Milstein, "Effects of radio propagation path loss on DS-CDMA cellular frequency reuse efficiency for the reverse channel," IEEE Trans. Veh. Technol., vol. 41, Aug. 1992.

[26] B. Sadeghi, V. Kanodia, A. Sabharwal, and E. Knightly, "Opportunistic media access for multirate ad hoc networks," in Proc. ACM MobiCom, Atlanta, GA, USA, Sept. 2002.

[27] G. Sharma, N. Shroff, and R. Mazumdar, "Joint congestion control and distributed scheduling for throughput guarantees in wireless networks," in Proc. IEEE International Conf. Computer Commun. (INFOCOM'07), Anocharage, AK, USA, May 2007.

[28] K. Tsudaka, M. Kawahara, A. Matsumoto, and H. Okada, "Power control routing for multi hop wireless ad-hoc network," in Proc. IEEE GLOBECOM, San Antonio, TX, USA, Nov. 2001.

[29] S. Wong, H. Yang, S. Lu, and V. Bharghavan, "Robust rate adaptation in 802.11 wireless networks," in Proc. ACM MobiCom, Los Angeles, CA, USA, Sept. 2006.

[30] Y. Xi and E. Yeh, "Distributed algorithms for spectrum allocation, power control, routing, and congestion control in wireless networks," in Proc. ACM MobiHoc, Montreal, QC, Canada, Sept. 2007. 